\title{
A survey of some problems in sterilization
}

\author{
ROBERT KNOX
}

From the Department of Bacteriology, Guy's Hospital Medical School, London

The methods of sterilization in use in many hospitals in Britain at the present time are inefficient and often unsafe and many so-called sterilizers do not sterilize. In recent years there has been increasing awareness of this unsatisfactory state of affairs. At first a few individuals condemned the situation and showed some glaring examples of breakdown in sterilization (Savage, 1944; Walter, 1948; Howie and Timbury, 1956; Bowie 1955, 1957). More recently, stimulated by the original observations, administrators and hospital authorities are becoming increasingly conscious that the whole machinery of sterilization in hospitals needs a drastic overhaul (Nuffield Provincial Hospitals Trust, 1958; Medical Research Council Working Party's Report, 1959). At the same time many new techniques and methods are becoming available. Rival claims are made for different methods and it is important to keep some sense of perspective and to decide for what purposes different methods of sterilization are most suitable. The aim of this article is to assess the present situation, to outline some of the main problems, and to suggest some of the lines along which development is likely to proceed in the immediate and in the more remote future.

\section{AVAILABLE METHODS OF STERILIZATION}

For some articles there are many methods of sterilization available but others can only be sterilized by perhaps one or at the most two different methods. The main methods are:

$1 a$, Dry heat: the conventional hot-air oven, conveyor belt methods, the conducting aluminium block, vacuum ovens; $b$, Moist heat: steam pressure sterilizers, downward-displacement type and highvacuum type.

2 Chemical methods: liquid disinfectants, gaseous disinfectants, especially ethylene oxide.

3 Irradiation by cobalt 60 or linear accelerator

There are, of course, many special methods, e.g., the use of ultraviolet light for sterilizing surfaces and for attempted sterilization of air, and the use of filtration for sterilizing fluids which cannot be subjected to other more drastic treatments. Special methods of filtration can also be used for the de- contamination or attempted sterilization of air. For surface disinfection formaldehyde may be satisfactory, provided it is used under proper conditions of humidity and temperature (Public Health Laboratory Service, 1958). With some types of equipment, e.g., bedpans, adequate disinfection may be achieved by exposure to steam at atmospheric pressure. Finally, for many purposes boiling is a perfectly satisfactory method, but it cannot be too strongly emphasized that many of these methods are only suitable in exceptional circumstances and none can be universally recommended as guaranteeing a sterile product.

GEOGRAPHICAL SCOPE AND LIMITATIONS OF DIFFERENT METHODS

One of the major problems to decide at the present time is to what extent sterilization should continue to be done on a purely local basis and to what extent it should be organized on a larger scale. Until very recently, sterilization has been a very local affair and no large British hospital so far as is known has had a complete central sterile service. Many new hospitals, however, have already constructed or are planning central sterile supply departments. A valuable account of the department already functioning at Musgrave Park, Northern Ireland, has been given by Allison (1960) who also discussed the development and planning of such a service. Surgeons and others in the past have been slow to accept the idea of central sterile supply. They have preferred to have their own instruments sterilized in the immediate neighbourhood of their own theatre. Syringes have been boiled up ready for use in the wards and so on. However, it is now generally agreed that central sterile supply has many advantages over purely local sterilization units scattered throughout a hospital. With the increasing complexity of modern sterilizing plant, it becomes more and more important to get the maximum use out of each unit. This alone is a powerful argument in favour of centralizing sterilization arrangements for any large hospital.

On the other hand there is an increasing feeling that many types of sterilization should not be carried 
out on a hospital basis at all but that commercial production and distribution would give more efficient and in the long run more economical results. This new approach is of course closely linked with the increasing production of disposable items of equipment, a trend to some extent forced upon us by the increasing cost of labour and the difficulty of getting efficient washing and sterilization equipment inside the hospital area. With certain types of equipment it looks as though the day of the large central sterile supply department in a hospital is already over before it has begun, and many hospital authorities must be wondering whether it is wise to sink a lot of money in the building of what in a few years' time may be a white elephant. The answer to many of these problems cannot be given at the present time and will come only as the result of experience. The best that can be done is to hope that experience will not be bought at too great a price. For example, it seems fair to say that the supply of disposable equipment sterilized by steam in commercial laboratories and distributed over a wide area is not likely to be an economic proposition. One reason is that the installation of large numbers of autoclaves with the amount of steam required to operate them efficiently is very expensive and the output is rather limited, whereas with other methods of sterilization, such as irradiation, although the capital cost is high, there is every prospect of being able to sterilize large quantities of material economically, for example, rubber gloves and syringes or needles. Of course it is unwise to generalize too much. The relative efficiency and cost of different methods must vary enormously with the nature of the material to be sterilized and with the purpose for which it is required.

\section{METHODS OF STERILIZATION}

STERILIZATION BY STEAM UNDER PRESSURE This is a field in which considerable advances have been made in the last few years, not so much because of the discovery of any new principles but because of an increasing realization that principles already well known should be efficiently applied if sterilization by steam is to yield the best possible results. It has been recognized for many years that rapid penetration of steam into the interior of dressings is essential if reliable sterilization is to be achieved. One of the traditional methods of removing air from steam sterilizers has been to make use of a steam ejector to draw out part of the air contained in the cylinder of the sterilizer before steam was admitted, and no sterilizer was regarded as efficient unless it had a 'vacuum' apparatus to remove air. It was, however, pointed out many years ago by
Underwood (1934), Savage (1937), and by others that this method even at its best gave only a poor vacuum and could not be expected to do more than remove two-thirds of the air originally present in the sterilizer. Often it was found that it was removing only about one-third of the air. This meant that a great deal of air was still left in the sterilizer. This interfered considerably with the penetration of steam and with the attainment of the required sterilizing temperature and was responsible for many sterilization failures. American opinion then began to stress the desirability of removing air not by this inefficient vacuum system but by an efficient system of downward displacement, and a great deal of American design and practice in hospitals was based on the assumption that the best way of removing air was by downward displacement (Perkins, 1956).

More recently attention has again been drawn to the need for rapid and efficient removal of air by means of the attainment of a really high vacuum, and, especially on the Continent, much progress has been made (Bowie, 1957).

A fresh investigation was made by Knox and Penikett (1958) of the degree of vacuum required in order that steam might penetrate efficiently into a standard drum situated in a particular position in an autoclave. The arbitrary figure of $20 \mathrm{~mm}$. or thereabouts was set as the order of vacuum which must be attained before admitting steam so as to ensure its rapid and reliable penetration. Further experimental data and a full account of this subject were given by Penikett (1960).

Knox and Penikett showed that in the conditions they used it was essential to reach this order of vacuum and emphasized that manufacturers must not be content with any machine which was not capable of rapidly producing it, and subsequent work has shown that with considerable variations in load and packing and with different sizes of sterilizers, this is the kind of vacuum which must be obtained in order to ensure uniformly reliable penetration of steam. The problem which then arose was how best this kind of vacuum could be obtained. Vacua of this order can be obtained by a number of different types of pump, for example, rotary oil pumps, water-ring pumps, and steam-jacketed pumps. Each of these has disadvantages and advantages but the problem has now become one of maintenance and engineering practice, and it is a question of finding out which method is going to give the best results over the longest period of time with the least maintenance trouble and the least cost to hospital authorities.

One point that is still unsettled is the exact time and temperature which should be used for sterilizing different types of equipment. There has been 
an unfortunate tendency to link together 'highvacuum' sterilizers with high-temperature, shorttime exposure to steam. It often seems to be taken for granted that the high-vacuum sterilizer should always be operated at a temperature of $134^{\circ} \mathrm{C}$. and at about $30 \mathrm{lb}$. steam pressure. This association is quite fallacious. There is no reason why conventional sterilizing times such as $121^{\circ} \mathrm{C}$. at 15 to $20 \mathrm{~min}$. should not be used with the high-vacuum sterilizer just as with the downward-displacement sterilizer. There may be certain advantages in higher temperatures and shorter times. But slight errors in timing become very important if only very short times are used for sterilization and it would seem that a greater margin of safety is present if periods of 15 to $20 \mathrm{~min}$. are used at temperatures of the order of 121 to $125^{\circ} \mathrm{C}$. rather than periods of only a minute or two at temperatures as high as $134^{\circ} \mathrm{C}$. This again is a question which can be answered only by future experience. At present opinion is divided. For example, we still have not enough information as to the correct time and temperature conditions for the efficient sterilization of rubber gloves by steam under pressure. Until very recently it was accepted that rubber gloves would not stand steam sterilization. Either the gloves were exposed to temperatures which could not possibly be expected to render them truly sterile or if they were exposed to sterilizing temperatures they were damaged beyond possibility of further use. It is now realized that a great deal of the damage to rubber gloves under these conditions was due to the long period of drying which was essential when the downwarddisplacement method was being used and that the damage was due to oxidation. If high-vacuum methods can be used and if drying conditions can rapidly be established by drawing a vacuum after the sterilization process is over, there seems to be no reason why rubber gloves should not be adequately sterilized either at high temperatures, such as $134^{\circ} \mathrm{C}$. for a very short period of time, say three to five minutes, or at temperatures of $121^{\circ}$ for 15 to $20 \mathrm{~min}$. Which of these is the better is still uncertain.

STERILIZATION BY DRY HEAT This subject is covered in another article and will not be referred to more than briefly here. Dry heat is used mainly for sterilizing syringes and certain instruments, especially those of which the cutting edge may be damaged by other methods. For syringes, one great advantage of dry heat is that they can be sterilized already assembled and can therefore be relied upon to be sterile inside the container in which they have been assembled so that the risk of contamination before an injection is given is reduced to the lowest possible level. In the conventional hot-air oven sterilization is carried out at a temperature of $160^{\circ} \mathrm{C}$. for one hour. Darmady and Brock (1954) have shown that conventional hot-air ovens may vary enormously in their performances and that there is considerable risk of either underheating or overheating, both of which are undesirable. In ovens fitted with efficient fans the temperature is much more uniformly regulated and for many purposes they are entirely satisfactory. Two recent developments are under trial at the present time in various forms of conveyor belt sterilizers and the solid aluminium block which heats the syringes placed in recesses inside the block by direct conduction. The conveyor belt seems to be the most useful apparatus for the large syringe service. The machine can be fed continuously with supplies of syringes, all of which can be guaranteed, provided that the temperature control is satisfactorily adjusted, to receive the same heat treatment as they are carried past the heating units. The aluminium block may perhaps have a considerable future for the small user. It is, of course, not so quick as a small autoclave which can indeed be used for sterilizing syringes but the autoclave has the strong disadvantage that if it is to be reliable then syringes must not be assembled, whereas the aluminium block has the great advantage of sterilizing assembled syringes which need no further handling until the injection is actually given. A syringe service using heat as the means of sterilization has to face competition from at least two other agents, namely, ethylene oxide and irradiation. More will be said about these in later sections. Here, it may be said that syringes sterilized by heat under proper conditions represent probably the most absolute standard of sterility which can be achieved. At the present time the same cannot be said of ethylene oxide except under very special conditions and even irradiation is not an infallible means of producing sterility. At the present time at least one commercial firm is running an efficient syringe service based on sterilization by heat from a series of gas ovens and making use of the conveyor belt principle.

IRRADIATION In recent years increasing attention has been paid to irradiation as a means of sterilizing articles of various kinds. This is being dealt with more fully in another article in this series, but here it may be useful to emphasize some main advantages of the method.

In Britain the method for which most information is available is the use of cobalt 60 as a source. Linear accelerators are also being developed for this purpose. The use of cobalt 60 is only suitable where the process can be carried out on a large enough scale to justify the capital cost and outlay involved. 
It must therefore be essentially an industrial process. If, however, this initial cost is accepted, the process has many advantages which would seem to make it almost unique. Articles of very diverse size, shape, and of different materials can be sterilized with complete reliability and very large quantities of material can be handled, giving probably a much greater capacity than that obtainable by any other method with comparable running costs. Materials such as syringes or rubber gloves can be assembled completely packaged and sterilized ready for use. With properly designed plant and flow of materials it can be guaranteed that all parts of the load and all items in it receive a sterilizing dose, and those problems of penetration and uniformity of treatment, which are so important in sterilization by steam and ethylene oxide, do not arise.

\section{GENERAL DISCUSSION}

Some of the outstanding problems in sterilization may perhaps best be discussed by posing a series of questions.

1 WHEN IS STERILIZATION NECESSARY? It is necessary here to steer a middle course between two extreme views. The first is that much of the recent interest in sterilization is misplaced and that simple measures such as boiling or the use of chemical disinfectants are perfectly satisfactory for most purposes. Those who hold this view believe that modern developments in sterilization are unnecessarily expensive and do not give an adequate return for the tremendous capital outlay involved. They are supported also by those who believe that central sterilizing services are bad for the morale of nurses and doctors and that it is better that they should be taught how not to sterilize properly rather than to rely on properly sterilized equipment. At the other extreme are those who demand that almost every article of equipment used in hospitals should te sterile. This certainly goes too far in the opposite direction and there is no doubt that there are many articles for which all that is needed is a reasonable standard of hygiene or cleanliness and not sterilization at all. Bedpans, Ryle's tubes, and even much of the water which is used in certain types of operative work might come under this heading. It might in fact be far safer to use good tap water for many of these purposes rather than socalled sterile water from an inefficient tank sterilizer which may be heavily contaminated with Proteus or Pseudomonas.

It would certainly make the task of hospital administrators a good deal easier if a decision could be reached as to what articles it is really necessary to sterilize. Once this decision is made then it seems clear that the best possible methods must be used for ensuring that they are properly sterilized and maintained in a sterile state. It is quite true that old-fashioned methods may suffice for many purposes and those who wish to retain them can often argue that no harm appears to have resulted from their use. One of the difficulties in all preventive medicine is that it is often impossible to trace any disasters that may occur if there is a breakdown in normal routine. On the other hand, there is certainly enough evidence to indicate that from time to time fatalities do occur as a result of breakdowns in sterilization. There is no refuting the argument that as far as surgical equipment is concerned it is our duty to see that those articles which really must be sterile are sterile when handed to the surgeon.

2 HOW SHOULD STERILIZATION BE DONE? Different types of equipment demand different methods of sterilization. For surgical dressings, packs, and many other fairly bulky items of equipment, steam under pressure is the most widely used and can be highly satisfactory. Here the problem is one of penetration of steam into materials from which it is important that the last trace of air should be removed. For this reason the pre-vacuum type of autoclave is very desirable. Autoclaves working by downward displacement of air can be effective and many which have not been working properly can be put into good working order (Howie and Timbury, 1956). Some of these if they have been manufactured to stand higher pressures and if they can satisfactorily pass insurance tests can be adapted for use as prevacuum sterilizers. But wherever new sterilizers are being installed it is desirable that pre-vacuum autoclaves should be specified. It has already been emphasized that these can be a good deal smaller than downward-displacement sterilizers intended to carry the same overall load (Medical Research Council Report 1960; Wells and Whitwell, 1960). The capital and running costs may therefore be much less than might be expected.

For emergency sterilization of instruments for use in theatre areas, there is no need for a high prevacuum sterilizer and a small downward-displacement type is useful. In these it is important that steam should enter very rapidly and air be removed quickly. Sterilizers of this sort, even though small, are best fitted with at least two discharge channels of adequate bore.

For the sterilization of bottled fluids, if it is not done on a commercial basis, a central sterile supply department needs a large downward-displacement sterilizer. The process is slow but devices are being 
developed to give more rapid cooling and shorten the whole cycle of sterilization.

For syringes, the standard of sterilization at present is by dry heat either in the form of a hot air oven or a conveyor belt or other devices which are being developed. Autoclaves may be used for sterilization of syringes but they are not so reliable or effective unless the syringe is dismantled and reassembled after sterilization. This is in itself a disadvantage and for that reason alone dry heat is preferable. Other methods such as the aluminium block and perhaps the pressure cooker have advantages for the small-scale user.

In the future, it seems that irradiation may well be the most satisfactory method of sterilizing syringes, especially if these are produced industrially and pre-packaged. The same applies to rubber gloves. These can of course be sterilized effectively by steam under pressure but disposable rubber gloves sterilized by irradiation are likely to be the answer to many of the special problems created by rubber goods. Irradiation on an industrial scale may also be used increasingly for many other articles.

It seems that there will still remain for some time many articles which cannot be sterilized by any of these methods, and for this type of equipment ethylene oxide is likely to be the most appropriate. The probable scope of ethylene oxide in the future is discussed by Kelsey (1961).

3 WHERE SHOULD STERILIZATION BE DONE? This raises many problems of organization and administration. The whole subject is in such a fluid state at the present moment that what is said today may be untrue tomorrow. As we move from the oldfashioned boiler in the ward or in the theatre to a system of centralized sterilization in a hospital, the logical conclusion is to move still further in the direction of large-scale production of pre-packaged sterile articles on a commercial baisis at competitive prices. But the question how far this process should go and what functions central sterile supply departments will have depends on future developments in the costs of materials, packaging, and transport, the ease with which supplies can be distributed at economic rates, and the area over which this can be done.

4 HOW OFTEN SHOULD ARTICLES BE STERILIZED? One of the dangers of sterilization which is not always realized is the fact that an article once sterile may gradually become contaminated if it is stored for any length of time, and the time necessary for contamination to occur is quite unknown in many cases. Any competent bacteriologist, however great his faith in the filtering effect of the conventional cottonwool plug, is rightly suspicious of 3 any sterile test-tubes with cottonwool plugs which have been lying about in his laboratory for weeks or months, especially if he has no means of being certain that the cottonwool plug has not at some time been removed and replaced. The extent to which contamination occurs in surgical dressings and other equipment depends on the wrapping and the container in which they are stored. This in itself is an important subject on which more work needs to be done.

5 WHAT IS MEANT BY STERILITY? This question is not so easy to answer as it seems at first sight. A few years ago most bacteriologists if asked what they meant by sterility would have been inclined to say that an object was either sterile or not. This is partly because when methods, such as sterilization by steam under pressure or adequate degrees of dry heat, are used there is usually such an enormous safety margin in the process that sterility can be guaranteed with almost complete certainty. On the other hand, with some of the newer methods of sterilization, such as ethylene oxide and rerhaps irradiation, it is becoming obvious that the achievement of sterility is largely a statistical process and that we can set our standard at several different levels. With irradiation or ethylene oxide, for example, we can aim at a kill of $10^{10}$ organisms or $10^{7}$ or $10^{5}$. It becomes therefore necessary to define sterility in terms of the attainment of a certain degree of killing and we have to make up our minds what sort of bacterial population we are likely to find in different types of material. There is little doubt that this argument also applies to steam though less obviously.

6 HOW DO WE KNOW WHEN AN ARTICLE IS STERILE? The whole question of proper sterility tests is complex. Practical and effective tests are not easy to devise. They fall into two main groups. In the first type we use a test organism whose resistance to the bactericidal agent is known and measure the number of organisms killed by certain doses or certain periods of exposure to the sterilizing agent. In the other type of test we simply take samples of the finished product and see whether or not they conform to certain accepted standards of sterility. The second kind of test is much more difficult to make reliable. In any laboratory there will always be a certain 'natural' contamination rate and it is not easy to decide, when a certain rate of contamination occurs, whether this represents the best that can be achieved with the existing technique even with articles which are theoretically sterile, or whether it represents a genuine breakdown in sterilization of the object under test. 
With certain types of equipment the interpretation of sterility tests is made even more difficult by the nature of the wrapping in which the material has been sterilized. Pre-packaged syringes, for example, are assembled and then placed inside an envelope of some plastic material which is then suitably sealed. The syringes are sterilized and issued ready for use inside these containers or envelopes usually made of materials which have to be cut by scissors or in some similar way. The very process of removing the syringe from its container in order to perform a sterility test is liable to cause contamination. How serious this risk is is still uncertain but it is obvious commonsense that, having taken great care to see that equipment is sterile, we must also ensure that the container can be opened with the least possible risk of contamination.

7 HOW SHOULD STERILIZERS BE TESTED? There are three main ways in which we can be assured whether a sterilizer is functioning efficiently or not.

i The sterilizer itself must have adequate instrumentation so that we can see whether the basic conditions necessary for its proper functioning are being achieved, for example, in the case of steam, temperature and pressure gauges, in the case of ethylene oxide, proper measurement of humidity and concentration of gas, and in the case of irradiation, accurate control of dosage.

ii For determining whether the necessary conditions have actually been reached in the material undergoing sterilization various kinds of tell-tale indicator are essential. The best-known example of these is the Browne's tube, which, although not absolutely ideal, gives information of great value and can be used in the day-to-day control of steam or dry-heat sterilization procedures. Indicators of this kind have the great advantage that they can be put in various positions in the sterilizer inside the loads undergoing sterilization. By preliminary experiment it is possible to find out whether there are any areas in the sterilizer or any parts of a load which are likely to escape adequate sterilization but the great advantage of the Browne's tube is that it can be used routinely if not inside every load at least inside a sample number of loads as a routine. The essential feature of an indicator of this kind is that it should be small. A number of new indicators are being developed and it is to be hoped that these will soon be available on a commercial basis for all the main methods of sterilization. It is of course possible in the case of steam to measure temperature actually inside loads by means of thermocouples or resistance thermometers, provided that these are small and can be moved about from one position to another. But clearly a small tube, such as the
Browne's tube, is much more useful for routine purposes provided it gives sufficiently reliable results.

iii Bacteriological tests can be carried out from time to time provided it is realized that they have some of the limitations referred to above and that sterility tests are not quite as easy to carry out as is sometimes thought. There is in fact considerable danger in occasional bacteriological tests being carried out in a hospital laboratory unless done by well-trained people who are well aware of the various pitfalls. Growth obtained from a test sample embedded inside a dressing pack may well indicate not a failure of sterilization at all but a failure in the technique employed in the laboratory. With some objects, especially if they are large, such failures are almost impossible to avoid, since it is extremely difficult to carry out an adequate sterility test on a large and bulky object without introducing some contamination in the process. On the other hand, the kind of sterility test which is often carried out in hospital laboratories can be even more dangerous in the opposite direction. For example, if proper controls are not used and if the organism used is too easily killed by heat, then the hospital pathologist may go on reporting sterile cultures till some disaster occurs. Only then does a thorough investigation reveal the fact that the sterilizer has not been functioning properly for a long time. In such cases the bacteriological tests are worse than useless as they give a false sense of security. Adequate inspection and control of the functioning of sterilizers are far more important than the purely routine multiplication of bacteriological tests which themselves may be quite uninformative. On the other hand, properly conducted bacteriological tests, preferably those in which test organisms of known resistance to the bactericidal agent are used, are the only direct method of telling whether the sterilizer is doing its job or not. The situation is very much the same as in the testing of water supplies. Bacteriological tests are the only tests which will tell us directly whether the bacterial content of a water supply conforms to certain standards but a single isolated test in inexpert hands is worse than useless. No bacteriological test is of any value at all, however expertly carried out, unless it is viewed in $\omega$ relation to the background of information about 2 the source from which the water comes, the way in 0 which it is treated, and the arrangements available $\mathbb{D}$ for its distribution and maintenance of its freedom? from contamination.

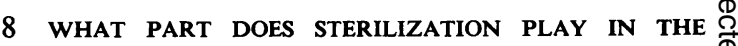
CONTROL OF HOSPITAL INFECTION? There is great $\stackrel{\unrhd}{\varrho}$ concern at the present time about the increasing 
frequency of staphylococcal and other infections in our hospitals (Williams, Blowers, Garrod, and Shooter, 1960). At the same time, as has been mentioned already, there have been many reports of breakdowns in sterilization and it is clear that failure to sterilize is widespread. It is natural that a connexion should have been suggested between hospital infection and breakdowns in sterilization. It has even been implied that if we could put our sterilization in order we should eliminate hospital infections or at any rate a large part of them. There is little to justify this attitude. Some infections which occur in hospitals are undoubtedly due to a breakdown in sterility: some cases of tetanus, for example, probably some cases of hepatitis, and an unknown number of other infections. But the staphylococcus which is causing most concern at the present time is quite easily killed by heat and there is not often enough evidence to suggest that a breakdown in sterilization is a major cause of staphylococcal outbreaks, though it may have been from time to time. Hospital infection spreads by many channels and efficient sterilization is only one of the ways by which its incidence may be reduced.

The true frequency of hospital infection is difficult to estimate but there is now general agreement that although the rate of post-operative sepsis is far lower than it was before the use of antibiotics, it is now a good deal higher than it was in the years immediately following their general introduction. Although in many ways surgical technique has made tremendous advances it is generally agreed that there is a real need for a tightening up of standards of operative and other techniques intended to reduce the risk of infection. One of these is the provision of sterile equipment. Here there is no doubt that standards have fallen to dangerously low levels. On these grounds alone it is essential that we should completely reorganize our sterilization procedures.

\section{CONCLUSION}

Two established methods of sterilization have stood up to the test of recent criticism, namely, the use of steam under pressure and dry heat. Other established methods such as boiling, steaming, and the use of liquid disinfectant are to be regarded no longer as methods of sterilization but rather as methods of disinfection. Of the newer methods, ethylene oxide and irradiation are the most promising. It is likely that for many years ethylene oxide will have a place for sterilizing certain special types of equipment, but though it is risky to prophesy it seems that irradiation is going to play the most useful part in the future. This inevitably means increasing centralization, increasing use of disposable equipment, and increasing industrialization of the whole process. We seem to be moving into an era in which sterilization will be much less a hospital responsibility but will be in fact an industrial process carried out by industrial methods and with adequate monitoring and methods of control on an industrial scale.

\section{REFERENCES}

Allison, V. D. (1960). Brit. med. J., 2, 772.

Bowie, J. H. (1955). Pharm. J., 174, 473, 489.

(1957). Hosp. Engng, 11, 74 and 98.

Darmady, E. M., and Brock, R. Barrington (1954). J. clin. Path., 7, 290.

Howie, J. W., and Timbury, M. C. (1956). Lancet, 2, 669.

Kelsey, J. C. (1961). J. clin. Path., 14.

Knox, R., and Penikett, E. J. K. (1958). Brit. med. J., 1, 680.

Medical Research Council Working Party's Report (1959). Lancet $1,425$. Ibid, 2, 1243 .

Nuffield Provincial Hospitals Trust (1958). Present Sterilizing Practice in Six Hospitals. London.

Penikett, E. J. K. (1960). Ph.D. thesis. London University.

Perkins, J. J. (1956). Principles and Methods of Sterilization. Thomas, Springfield, Illinois.

Public Health Laboratory Service (1958). Report of Committee on Formaldehyde Disinfection: Disinfection of fabrics with gaseous formaldehyde. J. Hyg. (Lond.). 56, 488.

Savage, R. M. (1937). Quart. J. Phar., 10, 445.

- (1944). Ibid., 17, 165.

Underwood, W. B. (1934). A Textbook of Sterilization. American Sterilizer Co., Erie, $\mathrm{Pa}$.

Walter, C. W. (1948). The Aseptic Treatment of Wounds. Macmillan, New York.

Wells, C., and Whitwell, F. R. (1960). Lancet, 2, 643.

Williams, R. E. O., Blowers, R., Garrod, L. P., and Shooter, R. A. (1960), Hospital Infection. Lloyd-Luke, London. 